\title{
Lässt sich das Screening in Risikopopulationen optimieren?
}

\author{
Die meisten Patienten mit Bronchialkarzinom befinden sich \\ zum Zeitpunkt der Diagnose bereits in einem \\ fortgeschrittenen Tumorstadium. Low-Dose-CTs sind für das \\ Screening zwar effektiv, dafür aber kostenintensiv. S. Spiro und \\ A. Hackshaw stellten nun eine neue Screening Strategie vor. \\ Thorax 2016; 71: 91-93
}

Eine US-amerikanische Studie konnte in der Vergangenheit zwar zeigen, dass sich mit regelmäßigen CT-Untersuchungen in einer unselektierten Population von Rauchern Bronchialkarzinome in einem früheren Stadium identifizieren ließen und so auch die Mortalität gesenkt werden konnte, allerdings erwies sich dies nicht als kosteneffektiv. Ähnliche Ergebnisse werden von einer holländisch-belgischen Studie erwartet. Daher wird es zukünftig wichtig sein, das CT effizienter einzusetzen oder Biomarker zu verwenden.

In der LungSEARCH-Studie wird dies versucht. Die Rationale dahinter ist, dass in einer Zielgruppe von Hochrisikopatienten die Detektionsrate von Karzinomen wahr- scheinlich höher sein wird und die Strategie somit kosteneffektiver werden dürfte. Ein solcher Risikofaktor ist die chronisch obstruktive Lungenerkrankung (COPD). Aufnahme in die Studie finden daher Patienten mit gering bis mäßig ausgeprägter COPD, die entweder aktuell rauchten (mindestens 20 Packyears und/oder 20 Jahre Raucheranamnese) oder ehemalige Raucher waren, die innerhalb der letzten 8 Jahre aufgehört hatten (ebenfalls mindestens 20 Packyears und/oder 20 Jahre Raucheranamnese). Die Teilnehmer werden randomisiert 2 Gruppen zugeordnet und unterziehen sich entweder 5 Jahre lang einem jährlichen Screening oder bilden Kontrollen ohne Intervention. Patienten der Interventionsgruppe geben ein- gangs Sputum ab. Bei auffälligem Befund bekommen sie die Möglichkeit zu jährlichen CT-Untersuchungen und Autofluoreszenz-Bronchographien für einen Zeitraum von maximal 5 Jahren. Bei normalem Sputum erfolgen jährliche Sputumkontrollen bis zu einem auffälligen Befund. Die Kontrollen werden in der Folge nicht mehr kontaktiert, sollten aber nach 5 Jahren eine Röntgenaufnahme des Thorax erhalten.

Fazit
Zwischen August 2007 und März 2011
wurden insgesamt 1568 Teilnehmer rek-
rutiert, davon 785 in der Interventions-
und 783 in der Kontrollgruppe. Bis April
2015 waren 65 Bronchialkarzinome re-
gistriert worden. Die initialen Ergebnisse
zeigen eine Inzidenz von abnormen Spu-
tumbefunden bei $23 \%$ der untersuchten
Teilnehmer im ersten und bei $15 \%$ im
zweiten Jahr. Sollte sich eine Verände-
rung des Tumorstadiums bei Diagnose
zugunsten der Interventionsgruppe erge-
ben, wollen die Autoren ihre Ergebnisse
möglicherweise in einer größeren Studie
bestätigen.

Dr. Johannes Weiß, Bad Kissingen

Ausgehend von diesen Ergebnissen untersuchten die Forscher am Modell, wie sich ein Fehlen des Eiweißes NFATc1 in spezifischen Abwehrzellen des Immunsystems auf die IL-9-Produktion auswirkt. Das Fazit: Im Modell wurde weniger des Botenstoffes IL-9 produziert, wodurch weniger IgE freigesetzt und dadurch weniger Histamin ausgeschüttet wurde. Folglich beeinflusst NFATc1 die allergische Komponente der Asthmaerkrankung. Die Erkenntnisse könnten dazu beitragen, neue Therapieansätze für allergisches Asthma bei Kindern zu finden. Die Studienergebnisse wurden kürzlich in der Zeitschrift Journal of Allergy and Clinical Immunology (DOI: 10.1016/j.jaci.2015.11.047) veröffentlicht.

Nach einer Mitteilung der FriedrichAlexander-Universität Erlangen-Nürnberg entscheidende Rolle bei der Entstehung der Erkrankung spielt. Die Wissenschaftler fanden heraus, dass Kinder mit Asthma im Alter zwischen 4 und 6 Jahren in den Abhöhte sen. Dies ist im Besonderen der Fall, wenn die Kinder zusätzlich zur Asthma-Erkrankung eine Allergie hatten, z. B. gegen Katzenhaare oder Hausstaubmilben. NFATc1 ist dafür bekannt, die Bildung des Botenstoffes Interleukin-9, kurz IL-9, zu fördern. Dieser Botenstoff wiederum kurbelt die Vermehrung spezieller Abwehrzellen an, die die Krankheit verschlimmert. Außerdem verstärkt IL-9 die Bildung des allergieauslösenden Immunglobulins E (IgE), was zur Ausschüttung entzündungsfördernder Stoffe wie bspw. Histamin führt, die das Asthma weiter begünstigen. 